\title{
The Expansion of RPE Atrophy after the Inverted ILM Flap Technique for a Chronic Large Macular Hole
}

\author{
Hisanori Imai Atsushi Azumi \\ Department of Ophthalmology, Kobe Kaisei Hospital, and Division of Ophthalmology, \\ Department of Organ Therapeutics, Kobe University Graduate School of Medicine, \\ Kobe, Japan
}

\section{Key Words}

Macular hole $\cdot$ Inverted internal limiting membrane flap technique $\cdot$ Chronic macular hole . Indocyanine green $\cdot$ Retinal pigment epithelium atrophy

\begin{abstract}
Purpose: To report a case of the expansion of submacular retinal pigment epithelium (RPE) atrophy after using the inverted internal limiting membrane (ILM) flap technique for a persisting, large, stage IV macular hole (MH). Case Report: A 79-year-old woman presented with a chronic large $\mathrm{MH}$ that remained open despite pars plana vitrectomy (PPV). The surgery was performed twice for the $\mathrm{MH}$ closure 14 years earlier. ILM peeling was not performed during the previous surgeries. The best-corrected visual acuity (BCVA) with the Landolt ring chart was 0.08 at her visit. The minimum MH diameter was $1,240 \mu \mathrm{m}$. Inverted ILM flap technique with $20 \%$ SF6 gas tamponade was performed for the MH closure. For the inverted ILM flap technique, 25-gauge PPV and ILM staining with indocyanine green were used. The ILM was peeled off for 2 disc diameters around the $M H$, but the ILM was not removed completely. The ILM was then inverted and covered the MH. Results: One month after surgery, the $\mathrm{MH}$ was closed, accompanied by glial cell proliferation spreading from the inverted ILM flap (as reported before). On the other hand, the area of the submacular RPE atrophy, which was already observed 1 week after surgery, gradually increased in size. BCVA improved to 0.3 six months after the surgery. Conclusions: The inverted ILM flap technique may be promising even for persisting large $\mathrm{MH}$ which were not closed in previous surgeries, but long-term observation is needed because the detailed behavior of the inverted ILM and the Müller cells after surgery is not yet known.

(c) 2014 S. Karger AG, Basel
\end{abstract}

Hisanori Imai, MD, PhD

Department of Ophthalmology, Kobe Kaisei Hospital

3-11-15 Shinoharakitamachi

Nada-ku, Kobe 657-0068 (Japan)

E-Mailimai@kobe-kaisei.org 
et al:: The Expansion of RPE Atrophy after the Inverted ILM Flap Technique for a Chronic Large Macular Hole

\section{Introduction}

Inverted internal limiting membrane (ILM) flap technique is a new method first reported by Michalewska and colleagues [1,2]. After their report, this technique was successfully applied for macular holes $(\mathrm{MH})$ with high myopia $[3,4]$. Here, we report a case of the expansion of the submacular retinal pigment epithelium (RPE) atrophy and gliosis on the surface of the retina after the inverted ILM flap technique was used for chronic large MH.

\section{Case Report}

A 79-year-old woman presented with a chronic large MH which remained open despite pars plana vitrectomy (PPV). The surgery for the MH closure was performed twice 14 years earlier. ILM peeling was not done during previous surgeries. The best-corrected visual acuity (BCVA) with the Landolt ring chart was 0.08 at her visit. The minimum MH diameter was $1,240 \mu \mathrm{m}$. Inverted ILM flap technique with $20 \%$ SF6 gas tamponade was performed for the MH closure. For the inverted ILM flap technique, 25-gauge PPV and ILM staining with indocyanine green (ICG) (0.25\%) were used. The ILM was peeled off for 2 disc diameters around the MH, but it was not completely removed. The ILM was then inverted and covered the $\mathrm{MH}$. One week after surgery, the $\mathrm{MH}$ was closed, accompanied by glial cell proliferation, spreading from the inverted ILM flap (as reported before) (fig. 1) [1]. On the other hand, the area of the submacular RPE atrophy and gliosis on the surface of the retina, which were already observed 1 week after surgery, gradually increased in size (fig. 1). BCVA improved to 0.3 six months after surgery.

\section{Discussion}

Michalewska et al. [1] reported that the inverted ILM flap technique stimulates proliferation of glial cells that fill $\mathrm{MH}$. In our case, the $\mathrm{MH}$ was closed successfully after 1 week and the photoreceptor defect decreased in diameter after 6 months. Finally, visual acuity improved from 0.08 to 0.3 . This result might support the hypothesis that proliferation of glial cells produces an environment for the photoreceptor to assume new positions in direct proximity to the fovea [1].

On the other hand, we observed the gradual expansion of RPE atrophy which was depicted as a hypoautofluorescent area on the fundus autofluorescein image (fig. 1). One possibility for this RPE atrophy might be the effect of ICG cytotoxicity. Several reports have demonstrated cytotoxicity of ICG to the RPE and neurosensory retina [5-8]. In our case, we used ICG for the ILM staining. It is possible that some quantity of ICG, which was left at the bottom of the MH and enclosed by the overlying ICG-stained ILM flap, provoked the RPE damage. Another possibility might be that several inflammatory cytokines secreted from the vitreous, like tissue necrosis growth factor- $\alpha[9,10]$, induced unexpected intensive Müller cell activation, followed by both RPE atrophy and gliosis.

\section{Conclusion}

In summary, the inverted ILM flap technique may be promising even for persisting large $\mathrm{MH}$ which were not closed in previous multiple surgeries. Long-term observation is needed 


\begin{tabular}{l|l}
\hline Case Rep Ophthalmol 2014;5:83-86 \\
\hline DOI: $10.1159 / 000360693$ & $\begin{array}{l}\text { C 2014 S. Karger AG, Basel } \\
\text { www.karger.com/cop }\end{array}$ \\
\hline
\end{tabular}

et al.: The Expansion of RPE Atrophy after the Inverted ILM Flap Technique for a Chronic Large Macular Hole

because detailed behavior of the inverted ILM and Müller cells after surgery is not yet known.

\section{References}

-1 Michalewska Z, Michalewski J, Adelman RA, Nawrocki J: Inverted internal limiting membrane flap technique for large macular holes. Ophthalmology 2010;117:2018-2025.

-2 Mahalingam P, Sambhav K: Surgical outcomes of inverted internal limiting membrane flap technique for large macular hole. Indian J Ophthalmol 2013;61:601-603.

-3 Kuriyama S, Hayashi H, Jingami Y, Kuramoto N, Akita J, Matsumoto M: Efficacy of inverted internal limiting membrane flap technique for the treatment of macular hole in high myopia. Am J Ophthalmol 2013;156:125-131.

4 Michalewska Z, Michalewski J, Dulczewska-Cichecka K, Nawrocki J: Inverted internal limiting membrane flap technique for surgical repair of myopic macular holes. Retina, 2013, Epub ahead of print.

-5 Maia M, Haller JA, Pieramici DJ, Margalit E, de Juan E Jr, Farah ME, Lakhanpal RR, Au Eong KG, Guven D, Humayun MS: Retinal pigment epithelial abnormalities after internal limiting membrane peeling guided by indocyanine green staining. Retina 2004;24:157-160.

6 Smiddy WE: The current status of macular hole surgery. Bull Soc Belge Ophtalmol 1996;262:31-42.

$\checkmark 7$ Rodrigues EB, Penha FM, de Paula Fiod Costa E, Maia M, Dib E, Moraes M Jr, Meyer CH, Magalhaes O Jr, Melo GB, Stefano V, Dias AB, Farah ME: The use of vital dyes in ocular surgery. Surv Ophthalmol 2009;54:576617.

-8 Burk SE, Da Mata AP, Snyder ME, Rosa RH Jr, Foster RE: Indocyanine green-assisted peeling of the retinal internal limiting membrane. Ophthalmology 2000;107:2010-2014.

-9 Caicedo A, Espinosa-Heidmann DG, Piña Y, Hernandez EP, Cousins SW: Blood-derived macrophages infiltrate the retina and activate Muller glial cells under experimental choroidal neovascularization. Exp Eye Res 2005;81:38-47.

10 Fernandez-Bueno I, Pastor JC, Gayoso MJ, Alcalde I, Garcia MT: Müller and macrophage-like cell interactions in an organotypic culture of porcine neuroretina. Mol Vis 2008;14:2148-2156. 


\begin{tabular}{l|l}
\hline Case Rep Ophthalmol 2014;5:83-86 \\
\hline DOI: 10.1159/000360693 & $\begin{array}{l}\text { @ 2014 S. Karger AG, Basel } \\
\text { www.karger.com/cop }\end{array}$ \\
\hline
\end{tabular}

et al.: The Expansion of RPE Atrophy after the Inverted ILM Flap Technique for a Chronic Large Macular Hole

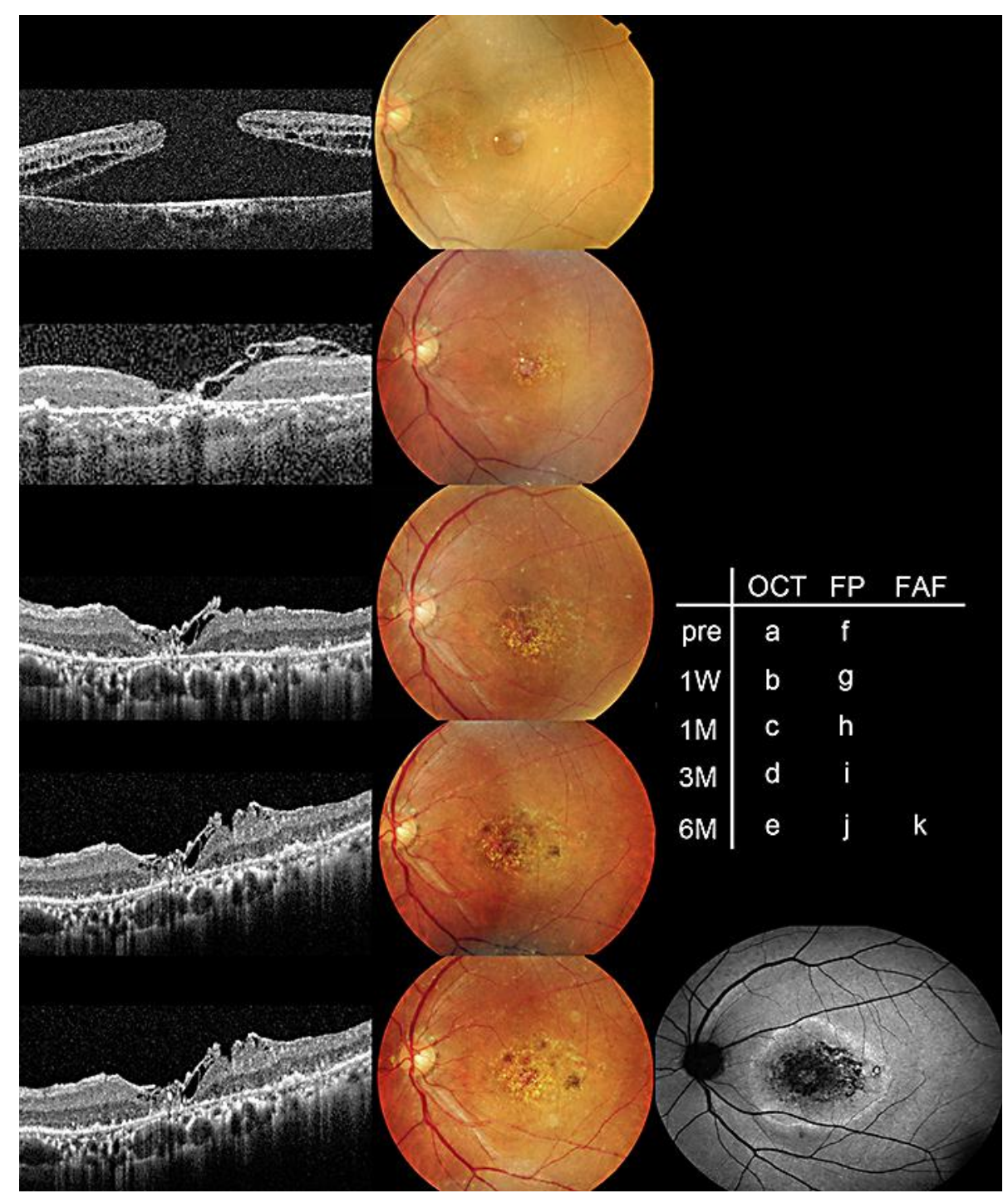

Fig. 1. The transition of funduscopic and optical coherence tomography (OCT) findings. The large MH (a, f) was closed 1 week after the inverted ILM flap technique (b, g). The photoreceptor defect gradually decreased in diameter (b-e). On the other hand, the area of the submacular RPE atrophy and gliosis on the surface of the retina, which was observed 1 week after surgery (g), expanded gradually (h-j). The area of the RPE atrophy was depicted as a hypoautofluorescent area on the fundus autofluorescein image 6 months after the procedure (k). FP = Fundus photography; FAF = fundus autofluorescein. 